\title{
Editorial Introduction to the Special Issue on Neuropsychological Assessment in Aging
}

\author{
Mathew J. Summers ${ }^{1}$ - Mark W. Bondi ${ }^{2,3}$
}

Received: 5 November 2017 / Accepted: 7 November 2017 / Published online: 18 November 2017

(C) Springer Science+Business Media, LLC, part of Springer Nature 2017

As the post-war 'baby boom' generation advances in age, a majority of countries are facing a health care crisis as the incidence of dementia rapidly increases (Alzheimer's Association, 2017). Many researchers are investigating potential biomarkers for early disease detection (Sperling et al. 2011) and trialing new drugs to halt neurodegenerative diseases (Hardy and De Strooper, 2017). However, there remains an ongoing debate relating to the best methods for detecting preclinical or prodromal changes in cognition associated with neurodegenerative diseases and differentiating these changes from the typical developmental changes of healthy aging (Snyder et al. 2014). An added complication to the detection of preclinical and clinical cognitive changes is the impact of secondary chronic diseases on cognition as well as ongoing generational changes in demographic factors, such as level of education, impacting on the sensitivity and psychometric properties of our neuropsychological measures of cognitive function.

While neuropsychologists undergo extensive training in neurocognitive evaluation with a strong background in the use and interpretation of cognitive and behavioural tests, increasingly tests of neuropsychological function are being employed by other professions without a strong understanding

Mathew J. Summers

msummers@usc.edu.au

1 Sunshine Coast Mind and Neuroscience - Thompson Institute, University of the Sunshine Coast, Sippy Downs, QLD, Australia

2 VA San Diego Healthcare System, San Diego, CA, USA

3 Department of Psychiatry, University of California San Diego, La Jolla, CA, USA of the psychometric properties of these tests. Measures designed to detect a clinical symptom with established cutpoints are increasingly being applied to detect preclinical symptoms. This clinical assessment strategy may be problematic when the test in use lacks evidence of sensitivity and specificity for the detection of preclinical or subclinical levels of impairment.

This special issue of Neuropsychology Review presents a series of systematic reviews to examine the current state of neuropsychological assessment in aging populations. The purpose of this special issue, including meta-analytic reviews, is to critically examine the current evidence relating to diagnostic sensitivity and specificity of neuropsychological assessment methods in neurodegenerative diseases, to highlight the need to consider other factors when assessing older adults, and to explore the evidence for the efficacy of cognitive interventions in older adults.

The first three papers in this special issue examine the diagnostic sensitivity and specificity of neuropsychological measures in detecting preclinical Alzheimer's disease (AD), mild cognitive impairment (MCI), and AD dementia, as well as predictive identification of individuals with $\mathrm{MCI}$ that progress to AD. Han and colleagues examine the evidence of neuropsychological changes in health older adults with in vivo biomarkers for $\mathrm{AD}$, finding that neuropsychological measures are sensitive to different stages of biomarker defined preclinical dementia. Belleville and colleagues examine the evidence that neuropsychological measures can identify adults with $\mathrm{MCI}$ who progress to $\mathrm{AD}$, reporting that multi-domain cognitive assessment displays the highest predictive accuracy for conversion to $\mathrm{AD}$. In the third paper in this series, Weissberger and colleagues examine the sensitivity and specificity of individual measures of memory in detecting $\mathrm{AD}$ and $\mathrm{MCI}$. The Weissberger analysis reveals high sensitivity and specificity for the detection of $\mathrm{AD}$, and lower but still useful sensitivity 
and specificity for MCI detection, highlighting the need for further test development in this latter area of practice.

An often overlooked factor in studies of aging-related neurodegenerative disease is the influence of secondary health conditions on primary disease detection. Typically, clinical studies exclude participants who display a secondary chronic disease, indicating that little information exists on the potential compounding influence of secondary diseases on the cognitive deficits exhibited. Cross and colleagues undertake a review of the evidence relating to a relatively common chronic disease, obstructive sleep apnoea (OSA), on cognitive function in healthy older adults. Their results indicating that OSA is an independent significant risk for cognitive deficits highlights the need for neuropsychological researchers to examine both the independent and combined effects of secondary diseases on cognitive function in older adults with aging-related neurodegenerative diseases.

The final two papers of this special issue examine the evidence for efficacy of cognitive interventions in older adults. Mewborn and colleagues undertake a review of randomized control trials (RCTs) of cognitive interventions in healthy older adults or adults with MCI. Similarly, Sherman and colleagues examine the RCT based evidence for efficacy of cognitive interventions on outcome in MCI. Mewborn and colleagues conclude that the evidence shows greater efficacy for cognitive interventions targeting working memory training, with Sherman and colleagues reporting that multicomponent or multi-domain interventions displaying the largest effect on cognitive outcome.

There are several important themes that emerge from this series of reviews. The existing collection of clinical neuropsychological tests demonstrate strong sensitivity and specificity for the detection of clinical disease states such as AD. However, these same measures lack sufficient sensitivity and specificity to detect subclinical or preclinical disease states when used in isolation. There is emerging evidence of the predictive diagnostic capacity of a multi-domain neuropsychological testing of subclinical and preclinical states such as MCI, with multi-domain approaches to assessment demonstrating strong diagnostic sensitivity and specificity. This evidence points both to the diagnostic importance of comprehensive neuropsychological assessment in detecting preclinical disease states in aging populations, as well as the need to develop new sensitive neuropsychological measures of subtle cognitive deficits (see Sperling et al. 2011). A related theme that emerges from these reviews is the need for further research to examine the independent effects of chronic disease states on cognitive function in ageing populations and to examine the contribution of chronic diseases to the cognitive profile of neurodegenerative diseases. An understanding of the contribution of underlying chronic diseases, such as OSA, Type II diabetes, and cardiovascular disease, to the cognitive profile of an older adult with an emerging neurodegenerative disease has significant implications for patient management and care.

The other important theme to emerge from the reviews relates to the efficacy of cognitive interventions in aging populations, with the evidence suggesting that multi-domain interventions display the greatest promise for effecting improved cognitive function in older adults. A consistent feature of the series of reviews and meta-analyses presented in this special issue is the paucity of high quality RCT studies. There is a clearly identified need for further research in aging populations covering typical age-related developmental changes as well as the identification of early changes in cognitive function associated with emerging diseases of aging, and neuropsychologists should take the lead in the design and implementation of high quality RCT studies in these areas.

Note from the Editors-in-Chief: Dr L.S. Miller acted as Associate Editor for the manuscript in this special issue authored by Drs. Stricker, Weissberger, Strong, Stefanidis, Summers and Bondi.

\section{References}

Alzheimer's Association. (2017). 2017 Alzheimer's disease facts and figures. Alzheimer's \& Dementia, 13(4), 325-373. https://doi.org/10. 1016/j.jalz.2017.02.001.

Hardy, J., \& De Strooper, B. (2017). Alzheimer's disease: Where next for anti-amyloid therapies? Brain, 140(4), 853-855. https://doi.org/10. 1093/brain/awx059.

Snyder, P. J., Kahle-Wrobleski, K., Brannan, S., Miller, D. S., Schindler, R. J., DeSanti, S., et al. (2014). Assessing cognition and function in Alzheimer's disease clinical trials: Do we have the right tools? Alzheimer's \& Dementia, 10(6), 853-860. https://doi.org/10.1016/ j.jalz.2014.07.158.

Sperling, R. A., Aisen, P. S., Beckett, L. A., Bennett, D. A., Craft, S., Fagan, A. M., et al. (2011). Toward defining the preclinical stages of Alzheimer's disease: Recommendations from the National Institute on Aging-Alzheimer's Association workgroups on diagnostic guidelines for Alzheimer's disease. Alzheimer's \& Dementia, 7(3), 280-292. 